\title{
Long-term anti-inflammatory diet in relation to improved breast cancer prognosis: a prospective cohort study
}

Kang Wang $\mathbb{i D}^{1,2}$, Jia-Zheng Sun $\mathbb{D i D}^{1,2}$, Qian-Xue Wu${ }^{1}$, Zhu-Yue $\mathrm{Li}^{3}$, Da-Xue $\mathrm{Li}^{4}$, Yong-Fu Xiong ${ }^{5}$, Guo-Chao Zhong ${ }^{6}$, Yang Shi ${ }^{7}$, Qing $\mathrm{Li}^{1}$, Jiali Zheng $\mathbb{1}^{8}$, Nitin Shivappa ${ }^{8,9,10}$, James R. Hébert ${ }^{8,9,10}$, Theodoros Foukakis ${ }^{11,12}$, Xiang Zhang ${ }^{1}$, Hong-Yuan Li $^{1}$, Ting-Xiu Xiang (iD $^{2 凶}$ and Guo-Sheng Ren (iD ${ }^{1,2 凶}$

Inflammation-modulating nutrients and inflammatory markers are established cancer risk factors, however, evidence regarding the association between post-diagnosis diet-associated inflammation and breast cancer survival is relatively sparse. We aimed to examine the association between post-diagnosis dietary inflammatory index (DII $\left.{ }^{\circledR}\right)$ and risks of all-cause and breast cancer-specific mortality. A total of 1064 female breast cancer survivors in the Prostate, Lung, Colorectal, and Ovarian Cancer Screening (PLCO) Trial prospective cohort, were included in this analysis if they had completed the diet history questionnaire (DHQ). Energy-adjusted DII $\left(\mathrm{E}-\mathrm{DII}{ }^{\mathrm{TM}}\right)$ scores were calculated based on food and supplement intake. Cox regression and competing risk models were used to estimate multivariable-adjusted hazards ratios (HRs) and $95 \%$ confidence intervals (95\% Cls) by E-DII tertile (T) for all-cause and breast cancer-specific mortality. With median follow-up of 14.6 years, there were $296(27.8 \%)$ deaths from all causes and 100 (9.4\%) breast cancer-specific death. The E-DIl was associated with all-cause mortality (HR T3 vs T1, 1.34; 95\% Cl, 1.01-1.81; $P$ trend, 0.049 , Table 2 ) and breast cancer mortality (HR T3 vs T1, 1.47; $95 \% \mathrm{Cl}, 0.89-2.43 ; P_{\text {trend, }} 0.13$; multivariable-adjusted HR for 1 -unit increment: 1.10; $95 \% \mathrm{Cl}: 1.00-1.22)$. Non-linear positive dose-response associations with mortality from all causes were identified for E-DII scores $\left(P_{\text {non-linearity }}<0.05\right)$. The post-diagnosis E-DIl was statistically significantly associated with mortality risk among breast cancer survivors. Long-term anti-inflammatory diet might be a means of improving survival of breast cancer survivors.

npj Breast Cancer (2020)6:36; https://doi.org/10.1038/s41523-020-00179-4

\section{INTRODUCTION}

Approximately 268,600 women were diagnosed with invasive breast cancer in the United States in 2019'. Breast cancer is rapidly becoming more common in other parts of the world ${ }^{2}$. In the past 40 years, breast cancer screening and treatments have improved substantially. Previous studies suggest that screening for early detection and improved treatment, including adjuvant therapy, have contributed to about one-half of the decline in breast cancer-related mortality ${ }^{3}$. Mortality can be lowered yet more by applying comprehensive disease management ${ }^{4}$. The importance of healthful diets for survivorship had been indicated in several studies ${ }^{5,6}$. However, debate continues about the best strategies for the dietary management of breast cancer patients to improve long-term survival ${ }^{7}$.

Chronic inflammation is implicated in breast cancer, and studies suggest a link between inflammation and breast cancer outcomes $^{8-11}$. More recently, literature has emerged that offers contradictory findings regarding the relationship between survival after a breast cancer diagnosis and specific nutrients known to modulate inflammation, such as dietary fat, fruits, vegetables, fiber, and alcohol ${ }^{12-15}$. The overall inflammatory potential of the diet may provide better insights into the effect of diet on breast cancer survival than assessing only a single nutrient; after all, a typical human diet consists of a variety of both proinflammatory and anti-inflammatory foods and nutrients ${ }^{16}$. The dietary inflammatory index $\left(\mathrm{DII}{ }^{\circledR}\right)$ reflects both a robust literature base and standardization of individual intakes to global referent values ${ }^{17}$. To date, a prospective cohort study from the Women's Health Initiative Study has used the DII to assess the association between post-diagnosis dietary inflammatory potential and survival of invasive breast cancer patients, indicating that consuming a more anti-inflammatory diet after breast cancer diagnosis reduced risk of death from cardiovascular disease ${ }^{18}$. Two retrospective cohort studies found inconsistent results: Jang et al. showed that antiinflammatory diets may decrease the risk of cancer recurrence and overall mortality in patients with breast cancer ${ }^{19}$, while findings from Italy did not suggest an association between the inflammatory potential of diet and the survival of female breast cancer patients ${ }^{20}$. Retrospective studies are susceptible to both selection and information biases. For example, it has been noted that positive dietary changes were more common among younger women or those who underwent chemotherapy ${ }^{21}$, and it is commonly assumed that dietary data are susceptible to biases associated with known outcomes, as in case-control studies ${ }^{22,23}$. Furthermore, some clinicopathological or lifestyle factors may mediate the association of diet and disease risk or survival, such as

\footnotetext{
${ }^{1}$ Department of the Endocrine and Breast Surgery, The First Affiliated hospital of Chongqing Medical University, Chongqing Medical University, Chongqing 400016, China. ${ }^{2}$ Key Laboratory of Molecular Oncology and Epigenetics, The First Affiliated Hospital of Chongqing Medical University, Chongqing, China. ${ }^{3}$ West China Hospital/West China School of Nursing, Sichuan University, Chengdu, China. ${ }^{4}$ Department of Breast Surgery, Chongqing Health Center for Women and Children, Chongqing 400000, China. ${ }^{5}$ The First Department of Hepatobiliary Surgery, Affiliated Hospital of North Sichuan Medical College, Nanchong 637007, China. ${ }^{6}$ Department of Hepatobiliary Surgery, The Second Affiliated Hospital of Chongqing Medical University, Chongqing, China. ${ }^{7}$ Division of Biostatistics and Data Science, Department of Population Health Sciences, Medical College of Georgia, Augusta University, Augusta, GA, USA. ${ }^{8}$ Department of Epidemiology and Biostatistics, Arnold School of Public Health, University of South Carolina, Columbia, SC 29208, USA.

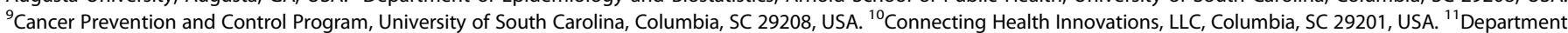
of Oncology-Pathology, Karolinska Institutet, 17164 Stockholm, Sweden. ${ }^{12}$ Breast Center, Theme Cancer, Karolinska University Hospital, 17176 Stockholm, Sweden.

凶email: xiangtx@cqmu.edu.cn; rengs726@126.com
} 
age $^{24}$, hormone receptor status ${ }^{25}$, body mass index (BMI) ${ }^{5,26}$, gene polymorphisms ${ }^{27}$, and smoking status ${ }^{28}$.

The energy-adjusted DII (E-DII ${ }^{\mathrm{TM}}$ ) is a composite measure of dietassociated inflammation which takes into account inflammatory effect of overall diet of an individual as opposed to looking at specific nutrients or food items. In this study, we aimed to comprehensively assess the association between inflammatory potential of post-diagnosis diet, as estimated by the E-DIl, and allcause and breast cancer-specific mortality among post-menopausal breast cancer patients from the Prostate, Lung, Colorectal, and Ovarian Cancer Screening Trial (PLCO) prospective cohort.

\section{RESULTS}

Patients characteristics

After a median follow-up time of 14.6 years from breast cancer diagnosis (interquartile range, 10.5-16.8 years), a total of 1064 eligible female breast cancer cases were available for analyses (Supplementary Fig. 1). Among them, there were 296 (27.8\%) all-cause deaths, and $100(9.4 \%)$ breast cancer-specific deaths. The 10-year overall survival rates were $87.3 \%, 86.6 \%$, and $80.7 \%$ in tertile (T) $1, \mathrm{~T} 2$, and $\mathrm{T} 3$ group, respectively (log-rank test, $P<0.001 ; \mathrm{HR}, 1.16 ; 95 \% \mathrm{Cl}$, 0.87-1.55 (T2 vs. T1), HR, 1.53; 95\% Cl, 1.15-2.03 (T3 vs. T1), Table 2 and Fig. 1a). Similarly, the 10-year breast cancer-specific survival rates were 93.2\%, 94.5, and 88.1\% in T1, T2, and T3 group, respectively (logrank test, $P<0.001 ; \mathrm{HR}, 1.76 ; 95 \% \mathrm{Cl}, 1.10-2.82$ (T2 vs. T1), HR, 1.70; $95 \% \mathrm{Cl}, 1.17-2.47$ (T3 vs. T1), Table 2 and Fig. 1b). As shown in Table 1, compared with cases with the most anti-inflammatory diets (i.e., -7.8 to -5.6 , E-DII T1), patients consuming the most pro-inflammatory diets (i.e., -4.1 to 4.9 , E-DII T3) had a higher total energy intake, higher $\mathrm{BMI}$, more current hormone therapy, and had lower education level, shorter follow-up time and time from breast cancer diagnosis to DHQ completion, and less aspirin use. The baseline characteristics of participants by tertile of E-DII from food, but without supplement, were shown as Supplementary Table 1.

Post-diagnosis dietary inflammatory index and survival of breast cancer patients

After the full adjustment for potential confounders, breast cancer patients consuming the most pro-inflammatory diets compared with the most anti-inflammatory diets had a 34\% higher risk of death from all-causes based on model2 (HR T3 vs T1, 1.34; $95 \% \mathrm{Cl}$, 1.01-1.81; $P_{\text {trend }}$ 0.049, Table 2; seen Supplementary Table 2 on E-DIl from food, but without supplement), where protective effect of an anti-inflammatory diet was also observed (HR for 1-unit increment: 1.06; 95\% Cl: 1.00-1.13, Table 2) when E-DIl was treated as a continuous variable. However, there was no statistically significant association between the E-DII and breast cancer-specific mortality in breast cancer patients in the multivariable-adjusted competing risk regression model (HR T3 vs T1, 1.47; $95 \% \mathrm{Cl}$, 0.89-2.43; $P_{\text {trend }}, 0.13$, Table 3; seen Supplementary Table 3 on E-DIl from food, but without supplement), we still found a protective effect of anti-inflammatory diet when considering E-DIl as a continuous variable (multivariable-adjusted HR for 1-unit increment: 1.10; $95 \%$ Cl: 1.00-1.22, Table 3). When we excluded cases with less than 1 year from breast cancer diagnosis to DHQ completion $(n=$ 454), both the HRs for all-cause mortality and breast cancer-specific mortality in model 2 became much stronger (for all-causes mortality, HR T3 vs T1 1.66; 95\% Cl,1.12-2.46, Table 2; for breast cancer-specific mortality, HR T3 vs T1 2.20; $95 \%$ Cl, 1.14-4.25, Table 3). The results of sensitivity analyses on 1-2 or $>2$ years before completing the DHQ were reported in Supplementary Table 4. Dose-response analyses found that E-DII scores were positively associated with risk of allcause mortality in a non-linear dose-response manner (Fig. 2).
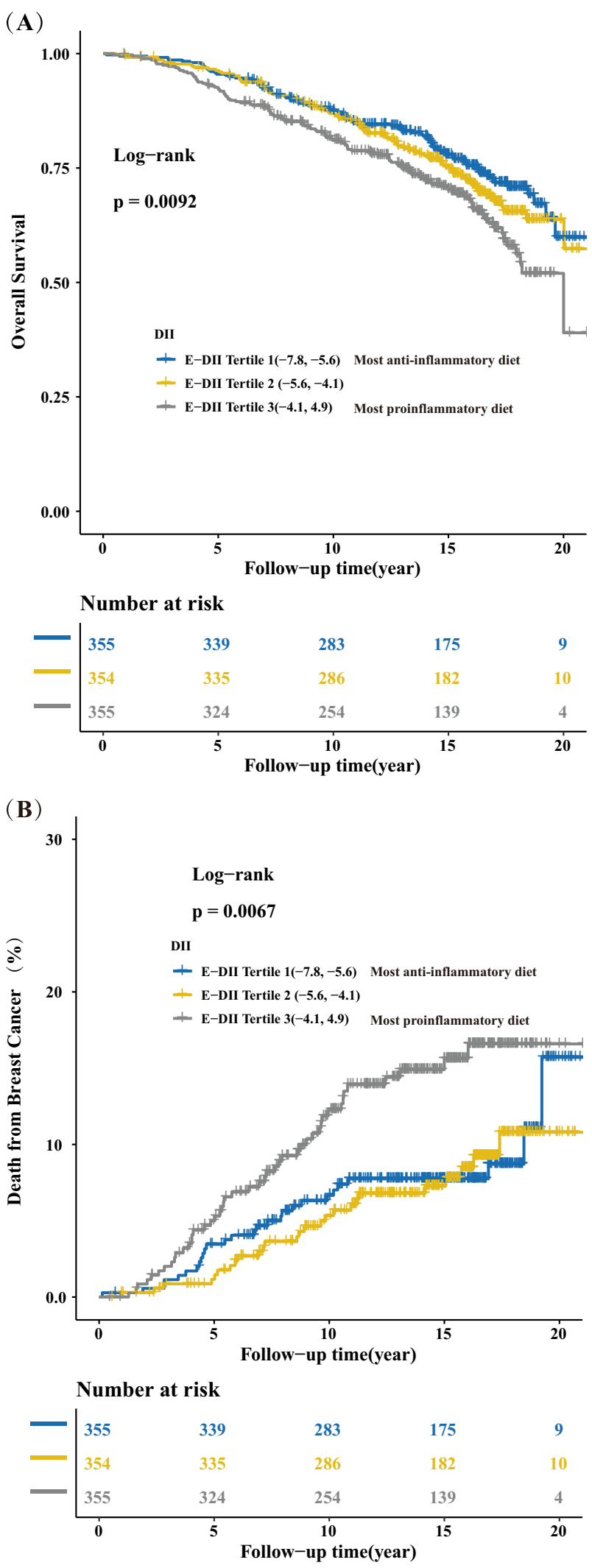

Fig. 1 Survival analysis between E-DIl scores in T1, T2, and T3. Kaplan-Meier curves of a overall survival; $\mathbf{b}$ breast cancer-specific mortality. 
Table 1. Baseline characteristics of 1064 breast cancer cases in the PLCO Cancer Screening Trial by tertile of E-DIl from food plus supplement.

\begin{tabular}{|c|c|c|c|c|}
\hline & \multicolumn{4}{|c|}{ Post-diagnostic exposure to dietary inflammatory potential } \\
\hline Number of cases & 355 & 354 & 355 & \\
\hline Age at breast cancer diagnosis (years) & $65(61,70)$ & $66(62,71)$ & $65(60.5,69.5)$ & $0.26^{\mathrm{a}}$ \\
\hline Total energy intake (kcal/day) & $1398.7(1084.2,1743.2)$ & $1410.6(1110.4,1792.7)$ & $1526.6(1189.2,1905.8)$ & $0.009^{\mathrm{a}}$ \\
\hline Alcohol intake (g/day) & $1.0(0,5.9)$ & $0.87(0,4.6)$ & $0.87(0,5.0)$ & $0.59^{\mathrm{a}}$ \\
\hline \multirow[t]{2}{*}{ Person-years of follow-up since breast cancer diagnosis } & $15.0(11.0,17.3)$ & $15.2(11.3,16.9)$ & $13.7(9.4,16.3)$ & $<0.001^{\mathrm{a}}$ \\
\hline & $N(\%)^{b}$ & $N(\%)^{\mathrm{b}}$ & $N(\%)^{b}$ & \\
\hline \multicolumn{5}{|l|}{ Trial arm } \\
\hline Intervention & $187(52.7)$ & $187(52.8)$ & $193(54.4)$ & $0.88^{\mathrm{b}}$ \\
\hline Control & $168(47.3)$ & $167(47.2)$ & $162(45.6)$ & \\
\hline \multicolumn{5}{|l|}{ Race/Ethnicity } \\
\hline Other $^{d}$ & $3(0.8)$ & $2(0.6)$ & $3(0.8)$ & \\
\hline \multicolumn{5}{|l|}{ BMI $\left(\mathrm{kg} / \mathrm{m}^{2}\right)$} \\
\hline$\leq 18.5$ & $1(0.3)$ & $3(0.8)$ & $3(0.8)$ & $<0.001^{\mathrm{C}}$ \\
\hline $18.6-25$ & $175(49.3)$ & $167(47.2)$ & $124(34.9)$ & \\
\hline $26-30$ & 119 (33.5) & $114(32.2)$ & $125(35.2)$ & \\
\hline $31-50$ & $60(16.9)$ & $70(19.8)$ & $103(29.0)$ & \\
\hline \multicolumn{5}{|l|}{ Marital status } \\
\hline Single $\mathrm{e}^{\mathrm{e}}$ & $18(5.1)$ & $11(3.1)$ & $15(4.2)$ & $0.83^{\mathrm{b}}$ \\
\hline Married or living as married & $252(71.0)$ & $260(73.4)$ & $247(69.6)$ & \\
\hline Divorced or separated & $38(10.7)$ & $39(11.0)$ & $41(11.5)$ & \\
\hline Widowed & $47(13.2)$ & $44(12.4)$ & $52(14.6)$ & \\
\hline$\$ 20,000-\$ 49,000$ & $149(42.0)$ & $143(40.4)$ & $171(48.2)$ & \\
\hline$\$ 50,000-\$ 99,000$ & $123(34.6)$ & $131(37.0)$ & $94(26.5)$ & \\
\hline$\$ 100,000-\$ 200,000$ & $28(7.9)$ & $21(5.9)$ & $23(6.5)$ & \\
\hline$<\$ 200,000$ & $3(0.8)$ & $4(1.1)$ & $4(1.1)$ & \\
\hline \multicolumn{5}{|l|}{ Smoking status } \\
\hline Never smoked & $181(51.0)$ & $196(55.4)$ & $187(52.7)$ & $0.005^{\mathrm{b}}$ \\
\hline Past smoker & $16(4.5)$ & $26(7.3)$ & $40(11.3)$ & \\
\hline Current smoker & $158(44.5)$ & $132(37.3)$ & $128(36.1)$ & \\
\hline \multicolumn{5}{|l|}{ Physical activity } \\
\hline Active less than one time per month & $48(13.5)$ & $40(11.3)$ & $52(14.6)$ & $0.41^{\mathrm{b}}$ \\
\hline Active at least one time per month & $307(86.5)$ & $314(88.7)$ & $303(85.4)$ & \\
\hline \multicolumn{5}{|l|}{ Hormone therapy } \\
\hline Never used & $112(31.5)$ & $109(30.8)$ & 139 (39.2) & $0.03^{b}$ \\
\hline Former used & $226(63.7)$ & $235(66.4)$ & $198(55.8)$ & \\
\hline Current used & $17(4.8)$ & $10(2.8)$ & $18(5.1)$ & \\
\hline \multicolumn{5}{|l|}{ Birth control pills } \\
\hline No & $151(42.5)$ & $171(48.3)$ & $167(47)$ & 0.27 \\
\hline Yes & $204(57.5)$ & $183(51.7)$ & $188(53.0)$ & \\
\hline \multicolumn{5}{|l|}{ Aspirin use } \\
\hline None & $99(27.9)$ & $77(21.8)$ & $110(31.0)$ & $0.01^{\mathrm{b}}$ \\
\hline$<$ Once/week & $74(20.8)$ & $81(22.9)$ & $91(25.6)$ & \\
\hline Once per week or more & $182(51.3)$ & $196(55.4)$ & $154(43.4)$ & \\
\hline
\end{tabular}




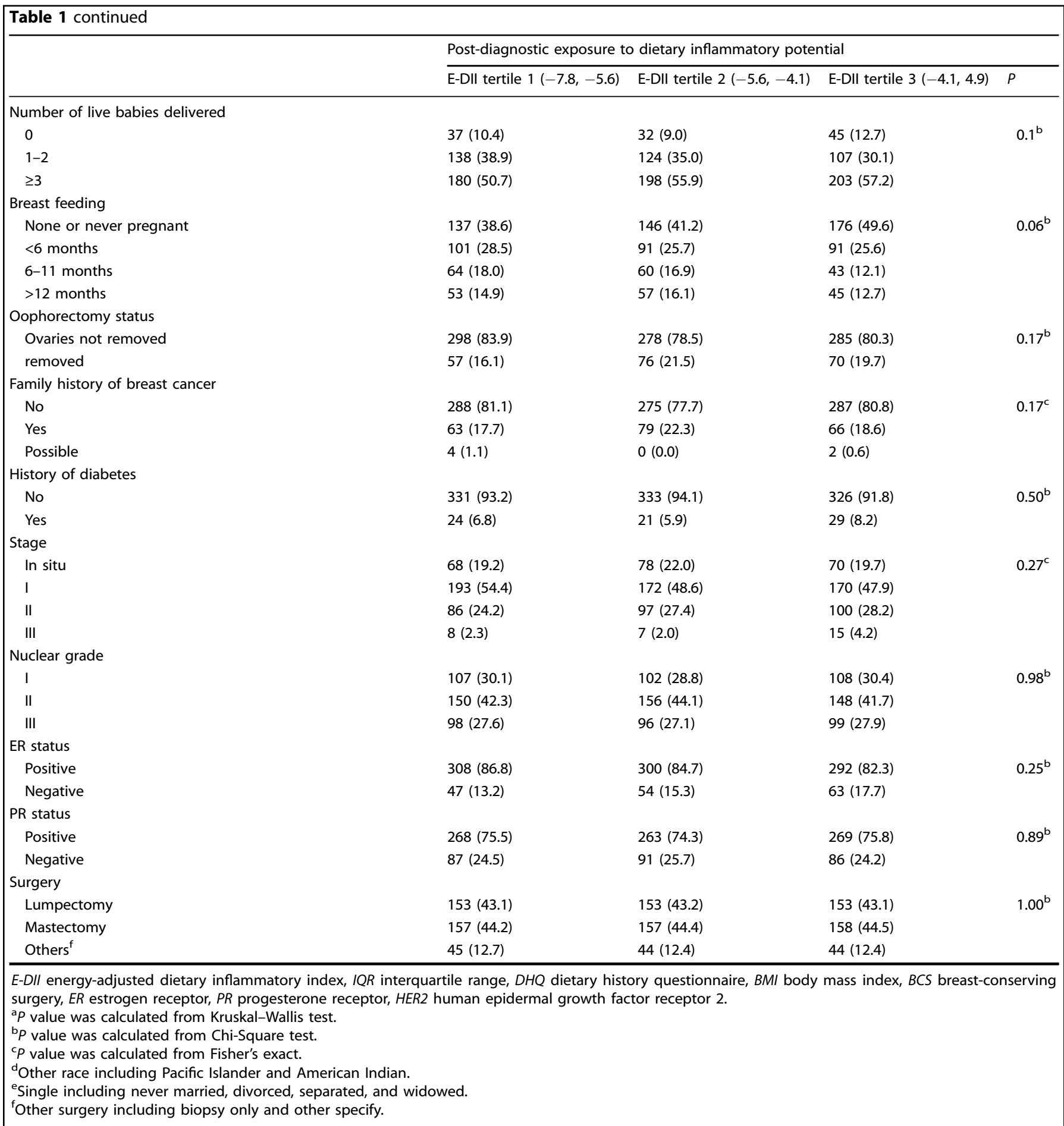

Stratified analysis

After stratifying by clinically relevant co-variables, the association between E-DII scores and deaths from all-causes differed only in the subgroups of follow-up time $\left(P_{\text {interaction }}<0.001\right)$. Women in the most pro-inflammatory diet (T3) subgroup with more than 15 person-years follow-up had a 2.18-fold higher risk of all-cause death than those with the most anti-inflammatory diet (HR T3 vs T1 2.18; $95 \% \mathrm{Cl}, 1.03-4.64 ; P_{\text {trend }}$ 0.03, Table 4); whereas no statistically association between E-DII and all-cause mortality was seen among cases with less than 15 person-years follow-up (HR T3 vs T1 $1.25 ; 95 \% \mathrm{Cl}, 0.91-1.72 ; P_{\text {trend }}, 0.18$, Table 4 ).

\section{DISCUSSION}

Overall, in this longitudinal analysis of post-menopausal breast cancer patients from the PLCO trial, a more anti-inflammatory diet after breast cancer diagnosis was associated with lower risks of both all-cause mortality and breast cancer-specific mortality. These risks varied by follow-up period, protective effects of consuming anti-inflammatory diet on prognosis of breast cancer became stronger when cases after long-term follow-up.

At present, only one prospective study has evaluated the influence of post-diagnosed dietary inflammatory potential on breast cancer outcomes ${ }^{18}$. In contrast to our findings, Zheng et al. 


\begin{tabular}{|c|c|c|c|c|}
\hline Tertile of E-DII score & Death from any cause $(n)$ & Person-years & \multicolumn{2}{|c|}{ Hazard ratio (95\% confidence interval) } \\
\hline Tertile $1(-7.8,-5.6)$ & 86 & 4942 & 1.00 (reference) & 1.00 (reference) \\
\hline Tertile $2(-5.6,-4.1)$ & 98 & 4888 & $1.14[0.85,1.52]$ & $1.16[0.87,1.56]$ \\
\hline Per 1-unit DIl increment & 296 & 14,307 & $1.10[1.04,1.17]$ & $1.06[1.00,1.13]$ \\
\hline \multicolumn{5}{|c|}{ After excluding cases with less 1 year from breast cancer diagnosis to DHQ completion ${ }^{c}$} \\
\hline Tertile $1(-7.8,-5.6)$ & 17 & 3400 & 1.00 (reference) & 1.00 (reference) \\
\hline Tertile $2(-5.6,-4.1)$ & 15 & 3280 & $0.89[0.45,1.76]$ & $0.99[0.73,2.05]$ \\
\hline Tertile $3(-4.1,4.9)$ & 25 & 2691 & $1.66[0.90,3.05]$ & $2.20[1.14,4.25]$ \\
\hline
\end{tabular}

\begin{tabular}{|c|c|c|c|c|}
\hline Tertile of E-DII score & Death from breast cancer $(n)$ & Person-years & \multicolumn{2}{|c|}{ Sub-distribution hazard ratio ( $95 \%$ confidence interval) } \\
\hline Tertile $1(-7.8,-5.6)$ & 28 & 4942 & 1.00 (reference) & 1.00 (reference) \\
\hline Tertile $2(-5.6,-4.1)$ & 26 & 4888 & $0.91[0.53,1.54]$ & $0.88[0.51,1.53]$ \\
\hline Per 1-unit DII increment & 100 & 14,307 & $1.16[1.06,1.27]$ & $1.10[1.00,1.22]$ \\
\hline \multicolumn{5}{|c|}{ After excluding cases with less 1 year from breast cancer diagnosis to DHQ completion ${ }^{c}$} \\
\hline Tertile $1(-7.8,-5.6)$ & 17 & 3400 & 1.00 (reference) & 1.00 (reference) \\
\hline Tertile $2(-5.6,-4.1)$ & 15 & 3280 & $0.89[0.45,1.76]$ & $0.99[4.73,2.05]$ \\
\hline Tertile $3(-4.1,4.9)$ & 25 & 2691 & $1.66[0.90,3.05]$ & $2.20[1.14,4.25]$ \\
\hline
\end{tabular}

found that among invasive breast cancer patients the E-DIl was associated with cardiovascular disease mortality rather than allcause or breast cancer-specific survival. That study had a slightly larger sample size but a shorter follow-up period than ours. Nevertheless, our study had a broader range of variability in the E-DII ( -7.8 to 4.9$)$ than theirs ( -6.8 to 3.8$)$. Another retrospective study found anti-inflammatory diets might improve the survival of breast cancer patients, particularly among younger women, those who were premenopausal, obese, had HR + breast cancer, had tumor more than $2 \mathrm{~cm}$, and had lymph node metastasis ${ }^{7}$. However, they used the DII (not the E-DII) and did not consider dietary supplements ${ }^{19}$. Three studies ${ }^{29-31}$ considered other cancer 

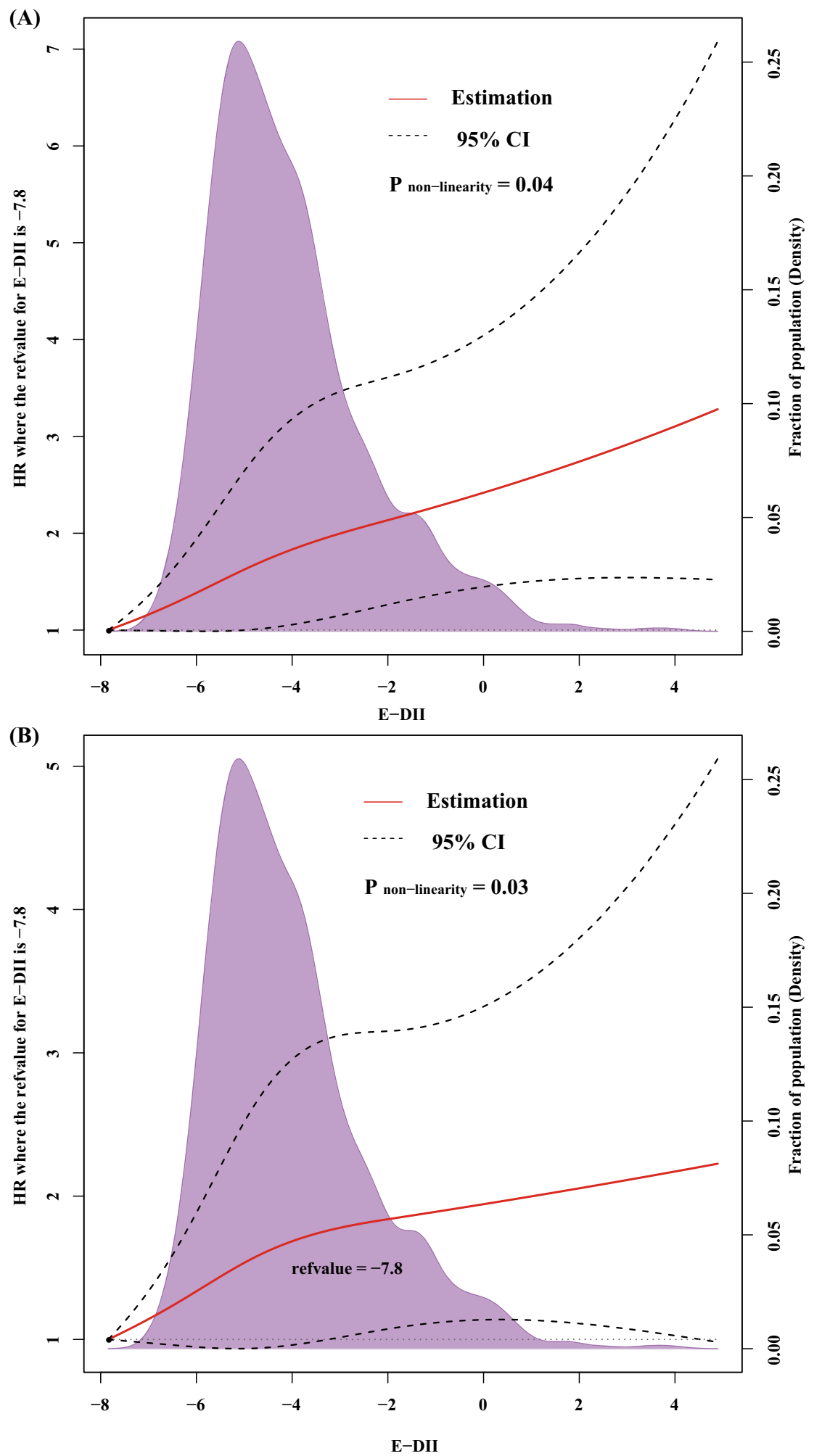

Fig. 2 Non-linear dose-response analysis on E-DII scores and all-causes mortality. Non-linear dose-response curves for a age and total energy-adjusted and $\mathbf{b}$ multivariable-adjusted Cox regression model. The reference level was set at -7.9 . A $P_{\text {non-linearity }}$ was obtained by testing the null hypothesis that regression coefficient of the second spline was equal to zero. $\mathrm{Cl}$ confidence interval.

types in relation to post-diagnosis E-DIl score on the risk of mortality, however, a post-diagnosis proinflammatory diet was not statistically significantly associated with mortality risk in cancer patients. Relatively short-term follow-up time and retrospective study designs maybe contribute to above-mentioned null results, which are similar to what we found when we examined the short duration ( $\leq 15$ person-years) subgroup. Further studies with extended follow-up time will be warranted in the future. 
Table 4. Risk of all-causes mortality stratified by follow-up time of breast cancer survivors across tertiles of post-diagnosis E-DII from food plus supplement in the PLCO Cancer Screening Trial.

\begin{tabular}{|c|c|c|c|c|c|}
\hline & E-DII tertile $1(-7.8,-5.6)$ & E-DII tertile $2(-5.6,-4.1)$ & E-DII tertile $3(-4.1,4.9)$ & $P_{\text {trend }}$ & $P_{\text {interaction }}{ }^{\mathrm{a}}$ \\
\hline$\leq 15$ person-years, $(n)$ & 180 & 172 & 216 & & $<0.001$ \\
\hline Death from any cause $(n)$ & 70 & 79 & 93 & & \\
\hline Multivariable-adjusted HR $(95 \% \mathrm{Cl})^{\mathrm{b}}$ & 1.00 (reference) & $1.21[0.87,1.69]$ & $1.25[0.91,1.72]$ & 0.18 & \\
\hline$>15$ person-years $(n)$ & 175 & 182 & 139 & & \\
\hline Multivariable-adjusted HR $(95 \% \mathrm{Cl})^{\mathrm{b}}$ & 1.00 (reference) & $1.24[0.61,2.54]$ & $2.18[1.03,4.64]$ & 0.04 & \\
\hline \multicolumn{6}{|c|}{ 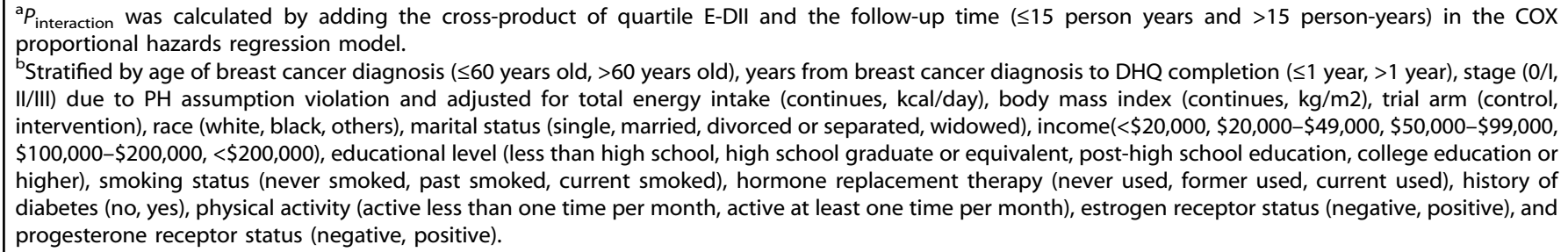 } \\
\hline
\end{tabular}

Prior studies have suggested that patients tend to make changes in their diet after a cancer diagnosis ${ }^{32,33}$. A previous study reported that dietary modifications were observed in women with breast cancer in Malaysia, and $66.7 \%$ were found to have decreased intake of energy, protein, total fat and vitamin $E$, and increased intake of carotene and vitamin $\mathrm{C}$ intake ${ }^{34}$. A prospective analysis from the Women's Health Initiative ${ }^{35}$ reported that dietary inflammatory potential before diagnosis is related to breast cancer death; however, future studies are needed to examine the inflammatory potential of post-diagnosis diet that is an important approach to conduct dietary intervention in process of the secondary breast cancer prevention. Improved recurrence-free survival and disease-free survival were observed in a large dietary intervention trial among women diagnosed with breast cancer who received dietary fat reduction treatment for breast cancer in the USA ${ }^{12}$. Furthermore, the Nurse's Health Study $(\mathrm{NHS})^{36}$ with a median of 9.3 years follow-up, including breast cancer patients more than 60 years old, investigated Dietary Approaches to Stop Hypertension (DASH) score and the Alternative Healthy Eating Index (AHEI)-2010 and risks of deaths from breast cancer and all causes. This NHS study reported better adherence to a priori dietary indices after breast cancer diagnosis was associated with a $28 \%$ (DASH) and 43\% (AHEI) reduced risk of non-breast cancer mortality. The WHI reported that in breast cancer survivors, postdiagnosis higher Healthy Eating Index (HEI)-2005 scores that reflected better quality diets were associated with better overall and cause-specific survival ${ }^{37}$. Because a lower E-DIl score, indicating a more anti-inflammatory diet pattern, is associated with better diet quality score (i.e., DASH, AHEI, and $\mathrm{HEI}$ ), our results are consistent with what was observed in those studies.

We did not find any interaction effects of ER/PR status on the association between E-DII score and risk of all-cause mortality, perhaps due to limited sample size and resulting loss in statistical power. A prior $\mathrm{WHI}$ study indicated that a positive relationship between E-DII scores and all-cause mortality risk was seen among ER-positive breast cancer cases and among the combined ERpositive and/or PR-positive cases but not ER-negative /PR-negative cases $^{38}$. In another WHI study, better dietary quality also was associated with a reduced risk of all-cause mortality among women with ER-positive tumors rather than ER-negative tumors ${ }^{37}$. Due to unbalanced distribution of the incidence rate and significantly different prognosis of ER-positive and ER-negative tumors $^{39}$, we suggest that further experimental and epidemiological studies are warranted to validate associations between diet and breast cancer subtypes.

We failed to show that the effect of the E-DIl on all-cause mortality in breast cancer survivors differed between smokers and non-smokers; a study focusing on high-grade serous ovarian carcinoma found a protective effect of anti-inflammatory diet on all-cause mortality risk among smokers ${ }^{31}$. Biologically, cigarette smoke contains many oxidants and free radicals and proinflammatory compounds that may activate endogenous mechanisms such as recruitment of neutrophils and macrophages to further increase the oxidant injury ${ }^{40}$. The shift in balance between oxidant/antioxidant in favor of oxidants, termed "oxidative stress", results in many pathological conditions including cancers ${ }^{41}$. Thus, anti-inflammatory diets, including supplements such as vitamins $(\mathrm{C}$ and $\mathrm{E})$, and $\beta$-carotene could protect smokers from experiencing oxidative stress ${ }^{41}$.

The strengths of this study include its prospective cohort design, the standardized dietary assessment using a FFQ that covered major foods and nutrients consumed by Americans, detailed covariables, long follow-up period, and the use of E-DIl that is a comprehensive assessment of dietary inflammatory from food plus supplement. Of note, this is the first study to indicate that a more anti-inflammatory diet after breast cancer diagnosis is associated with both better overall survival and breast cancer-specific survival. Sensitivity analyses and stratified analyses were conducted to highlight the stability of our results. Despite its strengths, several limitations should be noted. First, nearly $50 \%$ (2319 of 4561) of otherwise eligible women were excluded because they had a cancer diagnosis before completing the DHQ. In this process of exclusions, some selection bias might exist. Second, related to this first issue, the number of deaths was relatively small, thus precluding stratified analyses with sufficient statistical power to observe significant associations, especially in subgroups for breast cancer-specific mortality. We failed to detect a meaningful association between E-DII and the few observed cardiovascular disease mortality events, even though there is considerable evidence showing that consuming a more anti-inflammatory diet after breast cancer diagnosis can reduce the risk of death from cardiovascular disease ${ }^{18}$. Third, the FFQ in the PLCO provided only 35 out of 45 DIl components, which might lead to under- or overestimation of the relationship of E-DII with mortality ${ }^{17}$. In reality, we had demonstrated that missing even 15-20 parameters had little effect on DII scores in our prior work ${ }^{42}$. Fourth, the FFQ is known to 
suffer from a variety of biases associated with structured questionnaires. Most prominent among these are response sets such as social approval and social desirability ${ }^{43,44}$. This leads to the fifth weakness, which is that we had no information on any of these potentials in biasers ${ }^{45,46}$. Missing information on HER2 status and adherence to treatments resulted in missing data in the multivariable models, with the potential to bias HR estimates. Participants were excluded who did not have valid DHQ responses. This could potentially bias the results. When analyzing subjects who were excluded from the current analysis due to an invalid or missing $\mathrm{DHQ}$, we found that they were likely to be older, black, obese, divorced or widowed, have physical activity less than one time per month, and experience hormone therapy than those who were included in the risk estimates (Supplementary Table 5), indicating that the results of our study should be extrapolated to the US breast cancer survivors with caution.

In conclusion, this study evaluated the association of the inflammatory potential of diet with all-causes and breast cancerspecific mortality risks in a prospective cohort study of breast cancer survivors. Our findings support that anti-inflammatory potential of a post-diagnosis diet may be a means for reducing risk of breast cancer and all-causes death among breast cancer survivors. To precisely tailor the dietary interventions for breast cancer survivors in the future, additional well-designed cohort studies with large number of cases are warranted to validate our results and identify specific subgroups who would have a survival benefit of post-diagnosis anti-inflammatory diets.

\section{METHODS}

\section{Study design}

PLCO was sponsored by the National Cancer Institute $(\mathrm{NCl})$, conducted in the United States, and its purpose was to determine the effects of screening on cancer-related mortality and secondary endpoints ${ }^{47}$. In total, 154,897 eligible participants (76,682 males and 78,215 females), aged 55-74 years were enrolled from November 1993 to July $2001^{47}$. Participants were individually randomized to the intervention group or the control group in equal proportions. Data were collected on cancer diagnoses and deaths from all causes occurred through July 31, 2011.

\section{Ethics section}

All the participants provided written informed consent to participate in the study, and the study protocol (https://biometry.nci.nih.gov/cdas/ plco/) was approved by the Institutional Review Board of the United States National Cancer Institute ( $\mathrm{NCl})$.

\section{Study population}

To extract non-metastatic breast cancer patients who had valid DHQ responses after breast cancer diagnosis, our analytical cohort initially identified 4561 post-menopausal women who developed a first-primary breast cancer (International Classification of Diseases for Oncology, Third Edition, codes C50.0-C50.6, C508-C509). According to the exclusion criteria, we removed from consideration 2319 women whose dietary information were collected prior to their breast cancer diagnosis; 1017 cases with invalid DHQ responses (i.e., valid DHQ responses were defined as having DHQ completion date; alive at DHQ completion; <8 missing DHQ responses; and plausible caloric intake defined as within the sex-specific first and last percentiles of total energy); 14 cases who did not return baseline questionnaires and 147 cases with IV stage or unknown stage tumor. After these exclusions, the analytical cohort included 1064 breast cancer patients with follow-up data.

\section{Dietary assessment}

Dietary assessment was similar to our previous study ${ }^{48}$. Diet was assessed by a self-reported food frequency questionnaire (FFQ), the DHQ version 1.0 (National Cancer Institute, 2007) introduced in 1998 to the control and intervention arms within a median of three years after randomization in this trial ${ }^{49,50}$. On the $\mathrm{DHQ}$, participants reported their frequency of intake and portion size of 124 food items and supplement use over the previous year $^{50,51}$. Daily nutrient intake was calculated by the DietCalc software ${ }^{52}$, which integrated responses of food frequency, portion size, and other responses with a nutrient database based on national dietary data (USDA's 1994-96 Continuing Survey of Food Intakes by Individuals and supplemented by the Nutrition Data Systems for Research from the University of Minnesota $)^{49}$. The DHQ has been validated against four $24-\mathrm{h}$ dietary recalls (one in each season) among 1640 nationally representative participants in the Eating at America's Table Study where the energy-adjusted correlation coefficients for dietary factors ranged from 0.51 for vitamin $E$ to 0.78 for magnesium among women and from 0.41 for sodium to 0.83 for thiamin among men ${ }^{51}$.

\section{Energy-adjusted DII (E-DII ${ }^{\mathrm{TM}}$ ) score calculation}

The DII is a literature-derived, population-based index designed to estimate the overall inflammatory potential of an individual's diet. The details of the development of the DII have been published previously ${ }^{17}$. The energy-adjusted DII (E-DII) score was calculated based on reported nutrient and food intake from the DHQ which were linked to the corresponding inflammatory effect scores designated in the DII ${ }^{17,53}$ (Fig. 3 and Supplementary Fig. 2). Briefly, 1943 eligible peer-reviewed primary research articles incorporating cell culture, animal and epidemiological studies published up to 2010 on the effect of dietary factors on six inflammatory markers (interleukin (IL)-1 $\beta$, IL-4, IL-6, IL-10, tumor necrosis factor- alpha (TNF-a), and C-reactive protein (CRP)) were identified and scored to derive the component-specific inflammatory effect scores for 45 dietary factors (i.e., components of DII), which comprised macronutrients, micronutrients and some foods or bioactive components such as spices and tea ${ }^{17}$.

Eight DIl components including ginger, turmeric, garlic, oregano, rosemary, eugenol, saffron, and flavonols were not available from the DHQ. The remaining 37 components were available for E-DIl score calculation in our analysis. The food and nutrient consumption estimated at the DHQ was first adjusted for total energy per 1000 calories. To avoid the arbitrariness as a result of simply using raw intake amounts, the energyadjusted dietary intake was subsequently standardized to a composite dietary database representing energy-adjusted dietary intake from 11 populations living in different countries across the world ${ }^{17,54}$. The energyadjusted standardized dietary intake was then multiplied by the literaturederived inflammatory effect score for each DII component, and summed across all components to obtain the overall E-DII score ${ }^{17}$. Higher E-DII scores represent more pro-inflammatory diets while lower (i.e., more negative) E-DII scores indicate more anti-inflammatory diets. The DII score has been construct-validated with different dietary assessment instruments and found to be associated with higher levels of $\mathrm{IL}-6^{55}$, high-sensitivity $\mathrm{CRP}^{42}$, and homocysteine ${ }^{56}$. Because most of the participants $(79 \%)$ in the PLCO consumed supplements, and many dietary factors used in supplements have anti-inflammatory properties ${ }^{57}$, we report E-DII from food plus supplements.

\section{Covariate assessments}

Information on age at breast cancer diagnosis, race/ethnicity, marital status, education level, smoking status, income level, number of living birth, physical activity, breast feeding, oophorectomy status, family history of breast cancer and history of diabetes were assessed at baseline using self-administered questionnaires. Hormone therapy, birth control pills, aspirin use and total energy intake were acquired from DHQ. BMI was calculated as weight $(\mathrm{kg}) /$ height $(\mathrm{m})^{2}$ and categorized based on the World Health Organization criteria. Years from breast cancer diagnosis to DHQ completion was defined as interval time between breast cancer diagnosis and DHQ completion.

Detailed cancer characteristics such as diagnosis date, stage, tumor morphology (behavior, grade) and hormone receptor results were acquired. The cutoff for estrogen receptor (ER)-negative and progesterone receptor (PR)-negative IHC status was less than $1 \%$ staining in the nuclei.

\section{Ascertainment of death}

Outcomes included death from breast cancer and any cause. As previously described $^{58}$, death was primarily ascertained through a mailed annual study update questionnaire, with next of kin notifying the trial of death, which were verified by obtaining death certificates; searches of the National Death Index also were conducted to ascertain death. Autopsy and hospitalization records were used to determine the underlying cause of death. If these were unavailable, death certificates, medical records or other records were utilized. 


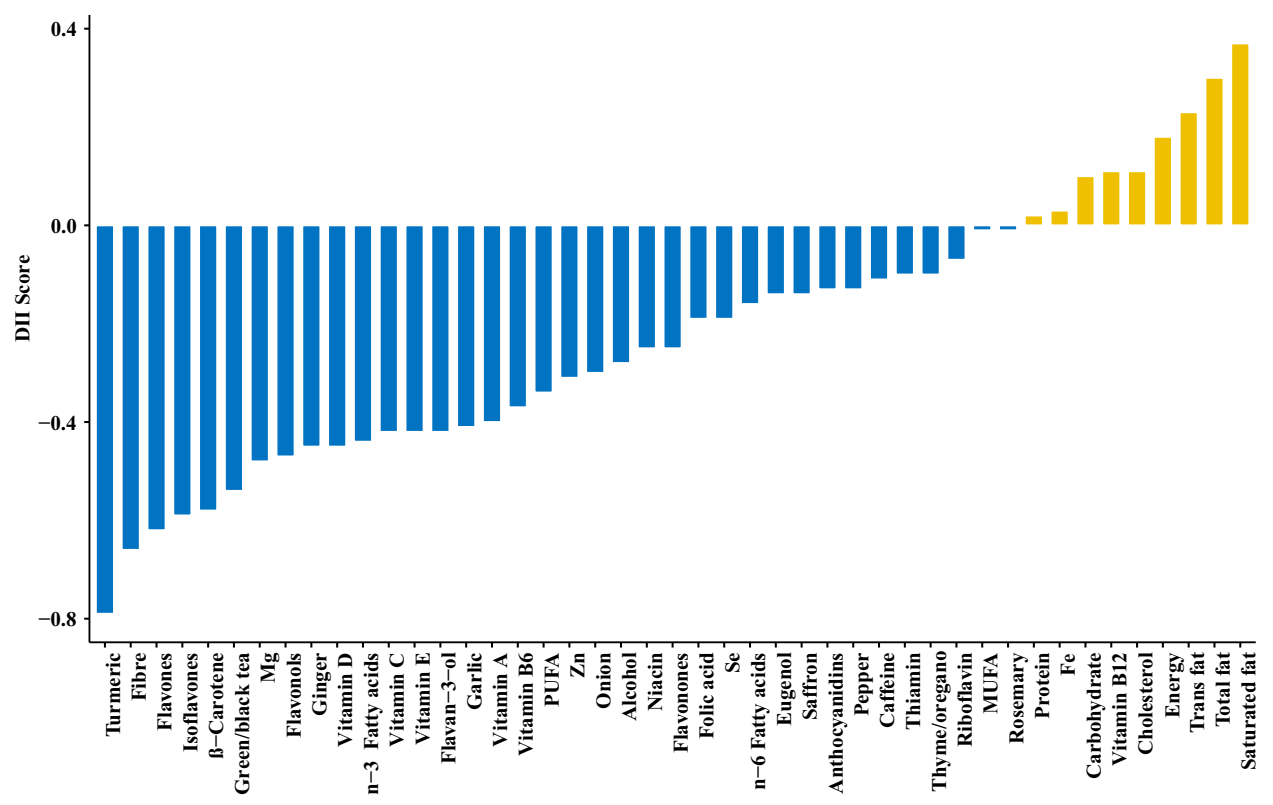

Fig. 3 Food parameter-specific DIl score. The DIl score distribution according anti-inflammatory and proinflammatory diets.

Ethical approval

All procedures performed in studies involving human participants were in accordance with the ethical standards of the institutional and/or national research committee and with the 1964 Helsinki declaration and its later amendments or comparable ethical standards.

\section{Informed consent}

Informed consent was obtained from all individual participants included in the PLCO study.

\section{Statistical analysis}

We divided the eligible patients into tertiles according to E-DII from food plus supplements with cutoff points determined from the distribution of the entire cohort. To present the baseline characteristics of the study cases, median ((interquartile range (IQR)) for continuous variables that are not normally distributed as indicated by Shapiro-Wilk normality test (all $P<0.05$ ) and frequencies (percentages) for categorical variables was calculated. Accordingly, Kruskal-Wallis test and Chi-Square test (Fisher's exact if needed) were employed to test differences of continuous and categoricalco-variates, respectively, between the three groups.

For each mortality outcome, women were followed from diagnosis of primary invasive breast cancer until death, loss to follow-up or the end of follow-up. Cox proportional modeling was fitted to estimate crude, age and total energy intake-adjusted and multivariable-adjusted HRs and $95 \%$ confidence intervals $(\mathrm{Cl})$ for breast cancer patients in the lowest E-DII tertile (most anti-inflammatory diet) as the referent. To minimize the potential impacts of competing risk bias on the association of E-DII with breast cancer-specific mortality risk, competing risk regression models were employed to estimate sub-distribution $\mathrm{HR}$ and $95 \% \mathrm{Cls}$, with non-breast cancer causes of death as competing risk events ${ }^{59}$. We tested a linear trend across tertile of E-DII using median E-DII value of each tertile, which was regarded as a continuous variable in regression analyses. Additionally, continuous E-DII variable was used to estimate risk estimates per 1-unit increment. Variables were considered as confounding factors if they were associated with both mortality risk and E-DII (in either continuous or categorical format) or they changed the crude risk estimate by $>10 \%$ in bivariate analyses ${ }^{60}$, in addition, prognostic factors for breast cancer patients from literature review also were treated as confounding factors. In model 1 , we adjusted for age at time of breast cancer diagnosis and total energy intake. Model 2 additionally adjusted for years from breast cancer diagnosis to DHQ completion, body mass index, trial arm, race, marital status, income, educational level, hormone replacement therapy, history of diabetes, physical activity, stage, and ER/PR status. Cancer stage and ER/PR status were used as substitutes for the currently unavailable cancer treatment data, as breast cancer stage and hormone receptor status may influence types of treatment received ${ }^{61,62}$. The proportional hazards (PH) assumption was examined using the Schoenfeld residual test ${ }^{63}$, and we only find that co-variables like age, years from breast cancer diagnosis to $\mathrm{DHQ}$ completion and stage violated the $\mathrm{PH}$ assumption in multivariable analyses. Thus, we fitted an extended Cox proportional hazards models stratified by age ( $\leq 60$ years old, $>60$ years old), years from breast cancer diagnosis to DHQ completion ( $\leq 1$ year, $>1$ year) and stage (0/I, II/III).

Effect modification by baseline characteristics and clinicopathological variables was examined by adding the cross-product of each effect modifier with E-DII tertile in the multivariable-adjusted model. Likelihood ratio tests were conducted, and $p$ values $<0.05$ were considered as an indicator of significant effect modification. We planned a priori stratified analysis by important co-variables that were considered clinically relevant, including age $(\leq 60,>60$ years), tumor stage (carcinoma in situ and invasive), smoking status (never smoker, former smoker, current smoker), $E R / P R$ status and follow-up time ( $\leq 15$ person-years, $>15$ person-years) whose median value is 15 person-years on the association between E-DII and all-cause mortality. Given the small number of breast cancer-specific death in this study, we did not further stratify analyses of breast cancerspecific mortality in interaction analyses.

We further assessed the potential non-linear dose-response relationship of E-DII to all-causes mortality through restricted cubic spline models with 3 knots at the 10th, 50th, and 90th percentiles ${ }^{64}$, and the reference level was set at -7.9 (the lowest value of E-DII in this study). Specifically, a $P_{\text {non- }}$ linearity was obtained by testing the null hypothesis that the regression coefficient of the second spline was equal to zero.

In sensitivity analyses, considering dietary changes due to adjuvant therapies involving chemotherapy and radiotherapy for the disease might affect appetite ${ }^{65}$, we excluded cases with less than 1 year from breast cancer diagnosis to DHQ completion.

All $P$ values reported are two-sided. Those less than 0.05 were considered statistically significant. All analyses were conducted using $\mathrm{R}$ software (version 3.4.1).

\section{Reporting summary}

Further information on research design is available in the Nature Research Reporting Summary linked to this article. 


\section{DATA AVAILABILITY}

Clinical and dietary data that support the findings of this study have been deposited in PLCO trial (https://biometry.nci.nih.gov/cdas/plco/), and PLCO has the following five ClinicalTrials.gov registration numbers: NCT00002540 (Prostate), NCT01696968 (Lung), NCT01696981 (Colorectal), NCT0169699 (Ovarian), and NCT00339495 (EEMS). A metadata record describing the underlying data, its availability and usage terms is available in the figshare repository: https://doi.org/10.6084/m9.figshare.12605924. ${ }^{66}$

\section{CODE AVAILABILITY}

The code for data processing and visualization is written in $\mathrm{R}$ and available upon request (K.W: kang_wang0822@hotmail.com).

Received: 12 March 2020; Accepted: 22 July 2020; Published online: 13 August 2020

\section{REFERENCES}

1. Miller, K. D. et al. Cancer treatment and survivorship statistics, 2019. CA Cancer J. Clin. 69, 363-385 (2019).

2. Goss, P. E. et al. Challenges to effective cancer control in China, India, and Russia. Lancet Oncol. 15, 489-538 (2014).

3. Berry, D. A. et al. Effect of screening and adjuvant therapy on mortality from breast cancer. N. Engl. J. Med. 353, 1784-1792 (2005).

4. Ma, J. et al. The American Cancer Society 2035 challenge goal on cancer mortality reduction. CA Cancer J. Clin. 69, 351-362 (2019).

5. Klassen, A. C. et al. "We're Just Not Prepared for Eating Over Our Whole Life": a mixed methods approach to understanding dietary behaviors among longer term cancer survivors. Integr. Cancer Ther. 17, 350-362 (2018).

6. Springfield, S., Odoms-Young, A., Tussing-Humphreys, L., Freels, S. \& Stolley, M. Adherence to American Cancer Society and American Institute of Cancer Research dietary guidelines in overweight African American breast cancer survivors. J. Cancer Survivorship Res. Pract. 13, 257-268 (2019).

7. Burden, S., Sremanakova, J., Jones, D. \& Todd, C. Dietary interventions for cancer survivors. Proc. Nutr. Soc. 1-11, https://doi.org/10.1017/S0029665118002690 (2018).

8. Dieli-Conwright, C. M. et al. Adipose tissue inflammation in breast cancer survivors: effects of a 16-week combined aerobic and resistance exercise training intervention. Breast Cancer Res. Treat. 168, 147-157 (2018).

9. Nagahashi, M. et al. Targeting the SphK1/S1P/S1PR1 axis that links obesity, chronic inflammation, and breast cancer metastasis. Cancer Res. 78, 1713-1725 (2018).

10. DeNardo, D. G. \& Coussens, L. M. Inflammation and breast cancer. Balancing immune response: crosstalk between adaptive and innate immune cells during breast cancer progression. Breast Cancer Res. BCR 9, 212 (2007).

11. Pierce, B. L. et al. Elevated biomarkers of inflammation are associated with reduced survival among breast cancer patients. J. Clin. Oncol. Off. J. Am. Soc. Clin. Oncol. 27, 3437-3444 (2009).

12. Chlebowski, R. T. et al. Dietary fat reduction and breast cancer outcome: interim efficacy results from the Women's Intervention Nutrition Study. J. Natl. Cancer Inst. 98, 1767-1776 (2006).

13. Pierce, J. P. et al. Influence of a diet very high in vegetables, fruit, and fiber and low in fat on prognosis following treatment for breast cancer: the Women's Healthy Eating and Living (WHEL) randomized trial. JAMA 298, 289-298 (2007).

14. McCullough, M. L. et al. Pre- and postdiagnostic diet in relation to mortality among breast cancer survivors in the CPS-II Nutrition Cohort. Cancer Causes Control. CCC 27, 1303-1314 (2016).

15. Chlebowski, R. T. et al. Low-fat dietary pattern and breast cancer mortality in the women's health initiative randomized controlled trial. J. Clin. Oncol. Off. J. Am. Soc. Clin. Oncol. 35, 2919-2926 (2017).

16. Krebs-Smith, S. M., Subar, A. F. \& Reedy, J. Examining dietary patterns in relation to chronic disease: matching measures and methods to questions of interest. Circulation 132, 790-793 (2015).

17. Shivappa, N., Steck, S. E., Hurley, T. G., Hussey, J. R. \& Hébert, J. R. Designing and developing a literature-derived, population-based dietary inflammatory index. Public Health Nutr. 17, 1689-1696 (2014).

18. Zheng, J. et al. Association between post-cancer diagnosis dietary inflammatory potential and mortality among invasive breast cancer survivors in the women's health initiative. Cancer Epidemiol. Biomark. Prev. Publ. Am. Assoc. Cancer Res. Cosponsored Am. Soc. Preventive Oncol. 27, 454-463 (2018).

19. Jang, H., Chung, M. S., Kang, S. S. \& Park, Y. Association between the dietary inflammatory index and risk for cancer recurrence and mortality among patients with breast cancer. Nutrients 10, https://doi.org/10.3390/nu10081095 (2018).
20. Zucchetto, A. et al. Dietary inflammatory index before diagnosis and survival in an Italian cohort of women with breast cancer. Br. J. Nutr. 117, 1456-1462 (2017).

21. Anderson, $C$. et al. Age- and treatment-related associations with health behavior change among breast cancer survivors. Breast 33, 1-7 (2017).

22. Li, D. et al. Dose-response relation between dietary inflammatory index and human cancer risk: evidence from 44 epidemiologic studies involving 1,082,092 participants. Am. J. Clin. Nutr. 107, 371-388 (2018).

23. $\mathrm{Ge}$, I. et al. Dietary inflammation potential and postmenopausal breast cancer risk in a German case-control study. Breast 24, 491-496 (2015).

24. Coughlin, S. S., Paxton, R. J., Moore, N., Stewart, J. L. \& Anglin, J. Survivorship issues in older breast cancer survivors. Breast Cancer Res. Treat. 174, 47-53 (2019).

25. Fung, T. T. et al. Diet quality is associated with the risk of estrogen receptornegative breast cancer in postmenopausal women. J. Nutr. 136, 466-472 (2006).

26. Mtintsilana, A. et al. Adiposity mediates the association between the dietary inflammatory index and markers of type 2 diabetes risk in middle-aged black South African women. Nutrients 11, https://doi.org/10.3390/nu11061246 (2019).

27. Yap, R. W. K., Shidoji, Y., Yap, W. S. \& Masaki, M. Association and interaction effect of AGTR1 and AGTR2 gene polymorphisms with dietary pattern on metabolic risk factors of cardiovascular disease in Malaysian adults. Nutrients $\mathbf{9}$, https://doi.org/ 10.3390/nu9080853 (2017).

28. Alkerwi, A. et al. Smoking status is inversely associated with overall diet quality: findings from the ORISCAV-LUX study. Clin. Nutr. 36, 1275-1282 (2017).

29. Ratjen, I. et al. Association between the dietary inflammatory index and all-cause mortality in colorectal cancer long-term survivors. Int. J. Cancer 144, 1292-1301 (2019).

30. Zucchetto, A. et al. Dietary inflammatory index and prostate cancer survival. Int. J. Cancer 139, 2398-2404 (2016).

31. Peres, L. C. et al. Prediagnostic proinflammatory dietary potential is associated with all-cause mortality among African-American women with high-grade serous ovarian carcinoma. J. Nutr. 149, 1606-1616 (2019).

32. Alfano, C. M. et al. Exercise and dietary change after diagnosis and cancer-related symptoms in long-term survivors of breast cancer: CALGB 79804. Psycho-Oncol. 18, 128-133 (2009).

33. Rabin, C. \& Pinto, B. Cancer-related beliefs and health behavior change among breast cancer survivors and their first-degree relatives. Psycho-Oncol. 15, 701-712 (2006).

34. Shaharudin, S. H., Sulaiman, S., Shahril, M. R., Emran, N. A. \& Akmal, S. N. Dietary changes among breast cancer patients in Malaysia. Cancer Nurs. 36, 131-138 (2013).

35. Tabung, F. K. et al. Association between dietary inflammatory potential and breast cancer incidence and death: results from the Women's Health Initiative. $B r$. J. Cancer 114, 1277-1285 (2016).

36. Izano, M. A., Fung, T. T., Chiuve, S. S., Hu, F. B. \& Holmes, M. D. Are diet quality scores after breast cancer diagnosis associated with improved breast cancer survival? Nutr. Cancer 65, 820-826 (2013).

37. George, S. M. et al. Better postdiagnosis diet quality is associated with reduced risk of death among postmenopausal women with invasive breast cancer in the women's health initiative. Cancer Epidemiol. Biomark. Prev. 23, 575-583 (2014).

38. J, Z. et al. Association between post-cancer diagnosis dietary inflammatory potential and mortality among invasive breast cancer survivors in the women's health initiative. Cancer Epidemiol. Biomark. Prev. Publ. Am. Assoc. Cancer Res. Cosponsored Am. Soc. Prevent. Oncol. 27, 454-463 (2018).

39. Bray, F. et al. Global cancer statistics 2018: GLOBOCAN estimates of incidence and mortality worldwide for 36 cancers in 185 countries. CA Cancer J. Clin. 68, 394-424 (2018).

40. Church, D. F. \& Pryor, W. A. Free-radical chemistry of cigarette smoke and its toxicological implications. Environ. Health Perspect. 64, 111-126 (1985).

41. Wang, M. et al. Lipid peroxidation-induced putative malondialdehyde-DNA adducts in human breast tissues. Cancer Epidemiol. Biomark. Prev. Publ. Am. Assoc. Cancer Res. Cosponsored Am. Soc. Prevent. Oncol. 5, 705-710 (1996).

42. Shivappa, N. et al. A population-based dietary inflammatory index predicts levels of C-reactive protein in the Seasonal Variation of Blood Cholesterol Study (SEASONS). Public Health Nutr. 17, 1825-1833 (2014).

43. Hebert, J. R., Clemow, L., Pbert, L., Ockene, I. S. \& Ockene, J. K. Social desirability bias in dietary self-report may compromise the validity of dietary intake measures. Int. J. Epidemiol. 24, 389-398 (1995).

44. Hebert, J. R. et al. Gender differences in social desirability and social approval bias in dietary self-report. Am. J. Epidemiol. 146, 1046-1055 (1997).

45. Hebert, J. R. et al. Systematic errors in middle-aged women's estimates of energy intake: comparing three self-report measures to total energy expenditure from doubly labeled water. Ann. Epidemiol. 12, 577-586 (2002).

46. Hebert, J. R. et al. Considering the value of dietary assessment data in informing nutrition-related health policy. Adv. Nutr. 5, 447-455 (2014).

47. Prorok, P. C. et al. Design of the prostate, lung, colorectal and ovarian (PLCO) cancer screening trial. Controlled Clin. Trials 21, 273s-309s (2000). 
48. Zheng, J. et al. Inflammatory potential of diet and risk of pancreatic cancer in the prostate, lung, colorectal and ovarian (PLCO) cancer screening trial. Int J. Cancer 142, 2461-2470 (2018)

49. Subar, A. F. et al. Evaluation of alternative approaches to assign nutrient values to food groups in food frequency questionnaires. Am. J. Epidemiol. 152, 279-286 (2000).

50. Diet History Questionnaire, Version 1.0. National Institutes of Health, Applied Research Program, National Cancer Institute. https://epi.grants.cancer.gov/DHQ/ about/ (2007).

51. Subar, A. F. et al. Comparative validation of the Block, Willett, and National Cancer Institute food frequency questionnaires: the Eating at America's Table Study. Am. J. Epidemiol. 154, 1089-1099 (2001).

52. NIH. Diet*Calc Analysis Program, Version 1.4.3. National Cancer Institute, Applied Research Program. https://epi.grants.cancer.gov/DHQ/database/ (2005).

53. N, S., SE, S., TG, H., JR, H. \& JR, H. Designing and developing a literature-derived population-based dietary inflammatory index. Public Health Nutr. 17, 1689-1696 (2014)

54. Wirth, M. D., Shivappa, N., Hurley, T. G. \& Hebert, J. R. Association between previously diagnosed circulatory conditions and a dietary inflammatory index. Nutr. Res. 36, 227-233 (2016).

55. Tabung, F. K. et al. Construct validation of the dietary inflammatory index among postmenopausal women. Ann. Epidemiol. 25, 398-405 (2015).

56. Shivappa, N. et al. Associations between dietary inflammatory index and inflammatory markers in the Asklepios Study. Br. J. Nutr. 113, 665-671 (2015)

57. Hamalainen, M., Nieminen, R., Vuorela, P., Heinonen, M. \& Moilanen, E. Antiinflammatory effects of flavonoids: genistein, kaempferol, quercetin, and daidzein inhibit STAT-1 and NF-kappaB activations, whereas flavone, isorhamnetin, naringenin, and pelargonidin inhibit only NF-kappaB activation along with their inhibitory effect on iNOS expression and NO production in activated macrophages. Mediators Inflamm. 2007, 45673 (2007).

58. Pierre-Victor, D. \& Pinsky, P. F. Association of nonadherence to cancer screening examinations with mortality from unrelated causes: a secondary analysis of the PLCO cancer screening trial. JAMA Intern. Med. 179, 196-203 (2019).

59. Lau, B., Cole, S. R. \& Gange, S. J. Competing risk regression models for epidemiologic data. Am. J. Epidemiol. 170, 244-256 (2009).

60. Maldonado, G. \& Greenland, S. Simulation study of confounder-selection strategies. Am. J. Epidemiol. 138, 923-936 (1993).

61. Fisher, B. et al. Treatment of lymph-node-negative, oestrogen-receptor-positive breast cancer: long-term findings from National Surgical Adjuvant Breast and Bowel Project randomised clinical trials. Lancet 364, 858-868 (2004).

62. Goldhirsch, A. et al. Meeting highlights: updated international expert consensus on the primary therapy of early breast cancer. J. Clin. Oncol. 21, 3357-3365 (2003).

63. Schoenfeld, D. Chi-squared goodness-of-fit tests for the proportional hazards regression model. Biometrika 67, 145-153 (1980).

64. Desquilbet, L. \& Mariotti, F. Dose-response analyses using restricted cubic spline functions in public health research. Stat. Med. 29, 1037-1057 (2010).

65. Fassier, P. et al. Modifications in dietary and alcohol intakes between before and after cancer diagnosis: results from the prospective population-based NutriNetSante cohort. Int. J. Cancer 141, 457-470 (2017).

66. Wang, K. et al. Metadata supporting the published article: Long-term antiinflammatory diet in relation to improved breast cancer prognosis: results from a prospective cohort study. Figshare. https://doi.org/10.6084/m9.figshare.12605924 (2020)

\section{ACKNOWLEDGEMENTS}

This research was approved and supported by contracts from the Division of Cancer Prevention, National Cancer Institute, National Institutes of Health, DHHS. The authors thank the Prostate, Lung, Colorectal, and Ovarian (PLCO) Cancer Screening Tria investigators and staff, and the authors acknowledge the PLCO study participants for their significant contributions to making this study possible.

\section{AUTHOR CONTRIBUTIONS}

K.W. conceived and designed the analysis, K.W., J.S., Q.W. and Z.L. collected the data N.S. and J.R.H. contributed E-DII data, Y.X., G.Z., D.L. and Y.S. performed the analysis, K.W. and X.Z. wrote the paper, J.R.H., T.F., H.L. T.X. and G.R. modified the draft. K.W., J.S., and Q.W. contributed equally to this work and should be considered as co-first authors.

\section{COMPETING INTERESTS}

J.R.H. owns controlling interest in Connecting Health Innovations LLC (CHI), a company that had licensed the right to his invention of the dietary inflammatory index $\left(\mathrm{DI} \mathrm{I}^{\circledast}\right)$ from the University of South Carolina in order to develop computer and smart phone applications for patient counseling and dietary intervention in clinical settings. N.S. is an employee of CHI.

\section{ADDITIONAL INFORMATION}

Supplementary information is available for this paper at https://doi.org/10.1038/ s41523-020-00179-4.

Correspondence and requests for materials should be addressed to T.-X.X. or G.-S.R.

Reprints and permission information is available at http://www.nature.com/ reprints

Publisher's note Springer Nature remains neutral with regard to jurisdictional claims in published maps and institutional affiliations.

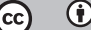

Open Access This article is licensed under a Creative Commons Attribution 4.0 International License, which permits use, sharing, adaptation, distribution and reproduction in any medium or format, as long as you give appropriate credit to the original author(s) and the source, provide a link to the Creative Commons license, and indicate if changes were made. The images or other third party material in this article are included in the article's Creative Commons license, unless indicated otherwise in a credit line to the material. If material is not included in the article's Creative Commons license and your intended use is not permitted by statutory regulation or exceeds the permitted use, you will need to obtain permission directly from the copyright holder. To view a copy of this license, visit http://creativecommons. org/licenses/by/4.0/.

(c) The Author(s) 2020 